\title{
BMJ Open Dose-response association between fluid overload and in-hospital mortality in critically ill patients: a multicentre, prospective, observational cohort study
}

\author{
Meiping Wang (D) , ${ }^{1,2}$ Bo Zhu, ${ }^{2}$ Li Jiang, ${ }^{2,3}$ Ying Wen, ${ }^{2,4}$ Bin Du, ${ }^{5}$ Wen Li, ${ }^{2}$ \\ Guangxu Liu, ${ }^{1}$ Wei Li, ${ }^{1}$ Jing Wen, ${ }^{1}$ Yan He, ${ }^{1}$ Xiuming $\mathrm{Xi}^{2}$
}

To cite: Wang M, Zhu B, Jiang L, et al. Dose-response association between fluid overload and in-hospital mortality in critically ill patients: a multicentre, prospective, observational cohort study. BMJ Open 2020;10:e039875. doi:10.1136/ bmjopen-2020-039875

- Prepublication history for this paper is available online. To view these files, please visit the journal online (http://dx.doi. org/10.1136/bmjopen-2020039875).

Received 29 April 2020 Revised 12 November 2020 Accepted 30 November 2020

Check for updates

(c) Author(s) (or their employer(s)) 2020. Re-use permitted under CC BY-NC. No commercial re-use. See rights and permissions. Published by BMJ.

For numbered affiliations see end of article.

Correspondence to

Professor Xiuming Xi; xixiuming2937@163.com and

Professor Yan He;

yanhe1220@126.com

\section{ABSTRACT}

Objectives Fluid management is important in ensuring haemodynamic stability in critically ill patients, but can easily lead to fluid overload (F0). However, the optimal fluid balance plot or range for critically ill patients is unknown. This study aimed to explore the dose-response relationship between FO and in-hospital mortality in critically ill patients.

Design Multicentre, prospective, observational study. Setting Eighteen intensive care units (ICUs) of 16 tertiary hospitals in China.

Participants Critically ill patients in the ICU for more than 3 days.

Primary outcome measures and analyses F0 was defined as the ratio of the cumulative fluid balance $(\mathrm{L})$ and initial body weight $(\mathrm{kg})$ on ICU admission, expressed as a percentage. Maximum F0 was defined as the peak value of FO during the first 3 days of ICU admission. Logistic regression models with restricted cubic splines were used to explore the pattern and magnitude of the association between maximum F0 and risk of in-hospital mortality. Age, sex, Acute Physiology and Chronic Health Evaluation II score, Sequential Organ Failure Assessment score on admission, main diagnosis on admission to ICU, comorbidities, time of maximum F0, mechanical ventilation, renal replacement therapy, use of vasopressors and centres were adjusted in multivariable analysis. Results A total of 3850 patients were included in the study, 929 (24.1\%) of whom died in the hospital. For each $1 \% \mathrm{~L} / \mathrm{kg}$ increase in maximum F0, the risk of in-hospital mortality increased by $4 \%$ (adjusted HR (aHR) 1.04, 95\% $\mathrm{Cl} 1.03$ to $1.05, \mathrm{p}<0.001$ ). A maximum F0 greater than $10 \%$ was associated with a $44 \%$ increased HR of inhospital mortality compared with an F0 less than 5\% (aHR $1.44,95 \% \mathrm{Cl} 1.27$ to 1.67). Notably, we found a non-linear dose-response association between maximum FO and in-hospital mortality.

Conclusions Both higher and negative fluid balance levels were associated with an increased risk of in-hospital mortality in critically ill patients.

Trial registration number ChiCTR-ECH-13003934.

\section{BACKGROUND}

Similar to mechanical ventilation (MV), renal replacement therapy (RRT) and use
Strengths and limitations of this study

- This large, multicentre, prospective cohort study was the first to comprehensively explore the nonlinear association between fluid overload and outcomes in critically ill patients.

- Multivariable logistic regression models with restricted cubic splines and propensity score matching method were applied.

- The observational design of the study does not allow the cause-and-effect relationship between fluid overload and outcomes to be inferred.

- Failure to consider fluid management prior to intensive care unit admission or in the operating theatre may underestimate the value of fluid overload.

of vasopressors, fluid management is an indispensable component of life support for critically ill patients. Optimal fluid resuscitation can ensure haemodynamic stability and improve tissue and organ perfusion, ${ }^{1}$ but frequently lead to fluid overload (FO).$^{23} \mathrm{FO}$ is associated with poor prognosis, including prolonged length of hospital stay and duration of $\mathrm{MV}^{4} \mathrm{FO}$ is also related to a higher mortality rate in critically ill patients. ${ }^{5-10}$

A majority of studies have defined FO as a cumulative fluid excess of $10 \%$ of body weight and found that it is an independent risk factor for mortality or other adverse events. ${ }^{511-13}$ A study on critically ill children showed that an FO greater than $5 \%$ was associated with a significantly increased risk of mortality and acute kidney injury (AKI). ${ }^{2}$ According to a study performed by the 'Dose Response Multicentre Investigation on Fluid Assessment (DoReMIFA)' group, any positive fluid balance is associated with an increased probability of death. ${ }^{14}$ However, all of those studies proposed a linear association between FO and outcomes, as a higher percentage of FO was related to an increased risk of adverse 
outcomes. This raised the question of whether there is an optimal volume plot or range of fluid balance for critically ill patients; knowledge on the dose-response association between $\mathrm{FO}$ and outcomes in critically ill patients remains limited. ${ }^{15}$

Using data from the China Critical Care Sepsis Trial (CCCST) study, we prospectively evaluated the pattern and magnitude of associations between maximum FO and in-hospital mortality and explored the optimal fluid balance for use in critically ill patients.

\section{METHODS}

\section{Patient and public involvement}

Patients or the general public were not involved in the design of the study.

\section{Study protocol and participants}

This study used data from the CCCST, a prospective, multicentre, observational cohort study where investigators examined the epidemiology of sepsis in critically ill patients at 18 intensive care units (ICUs) among 16 tertiary hospitals covering 7 geographical regions across China from 1 January 2014 to 31 August 2015. The study subjects included all adults (aged $\geq 18$ years) admitted consecutively to the ICU and stayed in the ICU for more than 3 days. For patients who had multiple admissions to the ICU, only the first ICU admission was considered. Patients who had missing fluid-related data were excluded. The study was registered at the Chinese Clinical Trials Registry (www.chictr.org.cn).

\section{Data collection}

Baseline data on demographic characteristics, diagnosis on admission, comorbidities, and clinical and laboratory values that were used to calculate illness severity scores, such as the Acute Physiology and Chronic Health Evaluation II (APACHE II) ${ }^{16}$ and the Sequential Organ Failure Assessment (SOFA) scores, ${ }^{17}$ were collected. Data on RRT, MV and use of vasopressors were continuously recorded for 7 days or until discharge from the ICU, whichever occurred earlier. Fluid balance was calculated daily. Dates of discharge from the ICU and the hospital were also documented. All these data were collected prospectively using a standard case report form and were then entered into the study's electronic database.

The primary outcome was in-hospital mortality. The secondary outcomes included in-ICU mortality and length of stay in the ICU and hospital.

\section{Management and missing data}

Less than $10 \%$ of clinical and laboratory data used to calculate the illness severity scores were missing in the whole cohort and were assumed to be of normal values and assigned a subscore of $0 .{ }^{18}$ Weight data were missing for $1.3 \%$ of patients, and a mean weight of $65 \mathrm{~kg}$ was inputted; $23(0.6 \%)$ patients had missing fluid-related values and were censored in the statistical analysis.

\section{Definition and calculation}

FO was defined as the ratio of cumulated fluid balance (CFB; in L) to body weight ( $\mathrm{kg}$ ) on initial ICU admission, expressed as a percentage. Maximum FO was defined as the peak value of FO during the first 3 days of ICU admission. Time of maximum FO was represented as the days between ICU admission and peak value of FO. Fluid intake included oral intake and intravenous fluid administration, and total fluid output included urine output, drained fluid, ultrafiltration fluid and estimated gastrointestinal losses. Insensible loss was not included in our study because it is difficult to assess. Shock was defined as a systolic blood pressure (SBP) $<90 \mathrm{~mm} \mathrm{Hg}$, mean arterial pressure (MAP) $<70 \mathrm{~mm} \mathrm{Hg}$, SBP decrease $\geq 40 \mathrm{~mm} \mathrm{Hg}$ or use of vasopressors to maintain tissue perfusion. Sepsis was defined as a life-threatening organ failure caused by infection within 48 hours on admission to the ICU according to the 'Surviving Sepsis Campaign: 2016'. ${ }^{19}$

\section{Statistical analysis}

Categorical variables are presented as numbers with percentages, and continuous variables are presented as mean \pm SD or median with IQR. The baseline characteristics of patients were compared using the $\chi^{2}$ test for categorical variables and the t-test, one-way analysis of variance or Wilcoxon rank-sum test for continuous variables.

The difference in CFB between the surviving and deceased groups during the first 3 days of ICU admission is presented in a box plot. The patients were divided into three groups according to the maximum $\mathrm{FO}$ value: maximum $\mathrm{FO}<5 \%, 5 \% \leq$ maximum $\mathrm{FO}<10 \%$, and maximum $\mathrm{FO} \geq 10 \%$. A Kaplan-Meier analysis was used to separately predict time to death in the hospital for the three maximum FO groups. Differences among the three groups were assessed using a log-rank test.

The association of maximum FO with in-hospital mortality was assessed using univariate and multivariable Cox proportional hazard regression models. The proportional hazard assumption for the Cox models was examined by including a time-dependent covariate with an interaction of maximum FO and a logarithmic function of survival time in the model. Separate models were performed to estimate maximum FO treated as a continuous variable (per $1 \% \mathrm{~L} / \mathrm{kg}$ increase) and a categorical variable $(<5 \%$ vs $<10 \%$ and $\geq 5 \%$ vs $\geq 10 \%)$. Analyses were first performed using a crude model (model 1), followed by three multivariable regression models. Model 2 was adjusted for age, sex, APACHE II score and SOFA score on admission. In model 3, we additionally adjusted for the main diagnosis on admission to ICU and comorbidities (respiratory disease, cardiovascular disease, hypertension, chronic renal dysfunction, tumour and no comorbidity). In model 4 , we additionally adjusted for time of maximum FO, MV, RRT and use of vasopressors. In models 2, 3 and 4, the centre was included as a random effect. Additionally, we further evaluated the pattern and magnitude of associations between maximum FO and in-hospital mortality using logistic regression models 
with restricted cubic splines (RCS) ${ }^{20}$ for the continuous maximum $\mathrm{FO}$ variable adjusting for all covariates (model 4). A maximum FO of 0 was treated as a reference, and four knots for the spline were placed at the 5 th, 35th, 65th and 95th percentiles of the maximum FO. Likelihood ratio test was used to assess whether there was a potential non-linear trend. In the sensitivity analysis, we explored the potential dose-response association of FO in patients with or without shock with risk of in-hospital mortality.

We used a propensity score matching (PSM) method to control for potential confounders. The propensity score was assigned based on the presence or absence of a maximum $\mathrm{FO} \geq 5 \% \mathrm{~L} / \mathrm{kg}$ and estimated using a multivariable logistic regression model. A 1:1 nearest neighbour matching algorithm was applied using a calliper width of 0.01 . The following variables were selected to generate the propensity score: age, sex, APACHE II score, SOFA score on admission, main diagnosis on admission to ICU, comorbidities (respiratory disease, cardiovascular disease, hypertension, chronic renal dysfunction, tumour and no comorbidity), MV, RRT and use of vasopressors.

A two-sided $p$ value of $<0.05$ was considered statistically significant. All analyses were conducted with IBM SPSS Statistics V.25.0 software for Windows and Stata V.15 statistical software.

\section{RESULTS}

\section{Clinical characteristics of the subjects}

Among the 4910 patients who were admitted consecutively, 3850 patients with their first 3 days of sequential fluid data were included in this study (figure 1$)$. The mean age was $61.8(18.1)$ years, $2501(65.0 \%)$ were male, and $929(24.1 \%)$ died in the hospital. Among the 3850 patients, $1882(48.9 \%)$ presented with a maximum FO $<5 \% \mathrm{~L} / \mathrm{kg}, 1030(26.8 \%)$ with a maximum $\mathrm{FO} \geq 5 \%$ and $<10 \% \mathrm{~L} / \mathrm{kg}$, and $938(24.3 \%)$ with a maximum $\mathrm{FO} \geq 10 \%$ $\mathrm{L} / \mathrm{kg}$. Compared with patients with a maximum FO less than $5 \%(\mathrm{~L} / \mathrm{kg})$, the other two groups of patients were more often male with higher illness severity scores and were likely to be diagnosed with sepsis, trauma and gastrointestinal conditions on admission to the ICU. They also needed more MV ( $64.3 \%$ vs $74.6 \%$ vs $84.0 \%$, $\mathrm{p}<0.001)$, RRT $(15.7 \%$ vs $18.1 \%$ vs $25.1 \%, \mathrm{p}<0.001)$

4910 eligible participants enrolled in the CCCST

1037 patients were excluded for ICU stays of less than 3 days

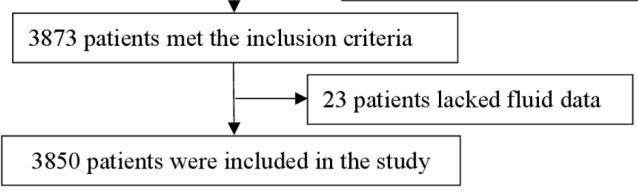

Figure 1 Flow chart of patients. CCCST, China Critical Care Sepsis Trial; ICU, intensive care unit. and vasopressors $(42.0 \%$ vs $50.2 \%$ vs $54.6 \%, \mathrm{p}<0.001)$ during the first 7 days of their ICU stay $(42.0 \%$ vs $50.2 \%$ vs $54.6 \%, \mathrm{p}<0.001$ ). These patients also had longer ICU and hospital stays (table 1 and online supplemental table S1).

\section{Association between $\mathrm{FO}$ and in-hospital mortality}

A progressive CFB was observed in both surviving and deceased patients since their ICU admission, and the two groups showed significantly different degrees of CFB at all time points (online supplemental figure S1). The maximum $\mathrm{FO}$ values were $6.5 \% \mathrm{~L} / \mathrm{kg}$ and $3.4 \% \mathrm{~L} / \mathrm{kg}$ in deceased and surviving patients, respectively (online supplemental figure S2). With an increase in maximum FO, in-hospital mortality increased from $17.5 \%$ to $35.5 \%$, and in-ICU mortality increased from $15.6 \%$ to $31.6 \%$ (table 1). The Kaplan-Meier analysis including the first 28 days of hospital stays revealed a significant survival benefit for patients with a maximum FO less than 5\% $\mathrm{L} / \mathrm{kg}(\mathrm{p}<0.001)$; patients with the highest percentage of maximum FO had the lowest survival rate (online supplemental figure S3).

In the multivariate Cox regression analysis of maximum FO, a 1.4-fold increase in the risk of in-hospital mortality was observed in the group with a maximum FO $>10 \% \mathrm{~L} /$ $\mathrm{kg}$ compared with the group with a maximum $\mathrm{FO}<5 \%$ $\mathrm{L} / \mathrm{kg}$ (HR 1.44, $95 \%$ CI 1.25 to $1.67, \mathrm{p}<0.001$; table 2 , model 4) after adjusting for potential confounders. When maximum FO was included as a continuous variable, maximum FO was significantly associated with in-hospital mortality (HR 1.04, 95\% CI 1.03 to 1.05, $\mathrm{p}<0.001$ ), regardless of potential confounders (table 2, model 4), indicating that for each $1 \% \mathrm{~L} / \mathrm{kg}$ increase in maximum FO the risk of in-hospital mortality increased by $4 \%$.

\section{Dose-response association between maximum F0 and in- hospital mortality}

The maximum FO during the first 3 days of ICU admission exhibited a skewed distribution, with a median of $3.9 \%$ (IQR 1.3-8.2\%) L/kg (figure 2). Using a multivariate logistic regression model with RCS, we observed a non-linear association between maximum FO and in-hospital mortality, and patients with a maximum FO of $2.4 \%$ $\mathrm{L} / \mathrm{kg}$ had the lowest risk of in-hospital mortality. Higher maximum $\mathrm{FO}$ values were significantly associated with an increased risk of in-hospital mortality, and patients with a maximum FO less than $0 \%$ exhibited a slightly increased risk of in-hospital mortality (figure 3A). A similar curve was observed when we explored the potential effects of maximum FO on the risk of in-hospital mortality in patients with or without shock in the sensitivity analysis (figure 4). It was found that a maximum FO greater than $9.6 \% \mathrm{~L} / \mathrm{kg}$ was an independent risk factor in patients with shock (figure 4A); however, in patients without shock, this value was $6.3 \% \mathrm{~L} / \mathrm{kg}$ (figure $4 \mathrm{~B}$ ). 


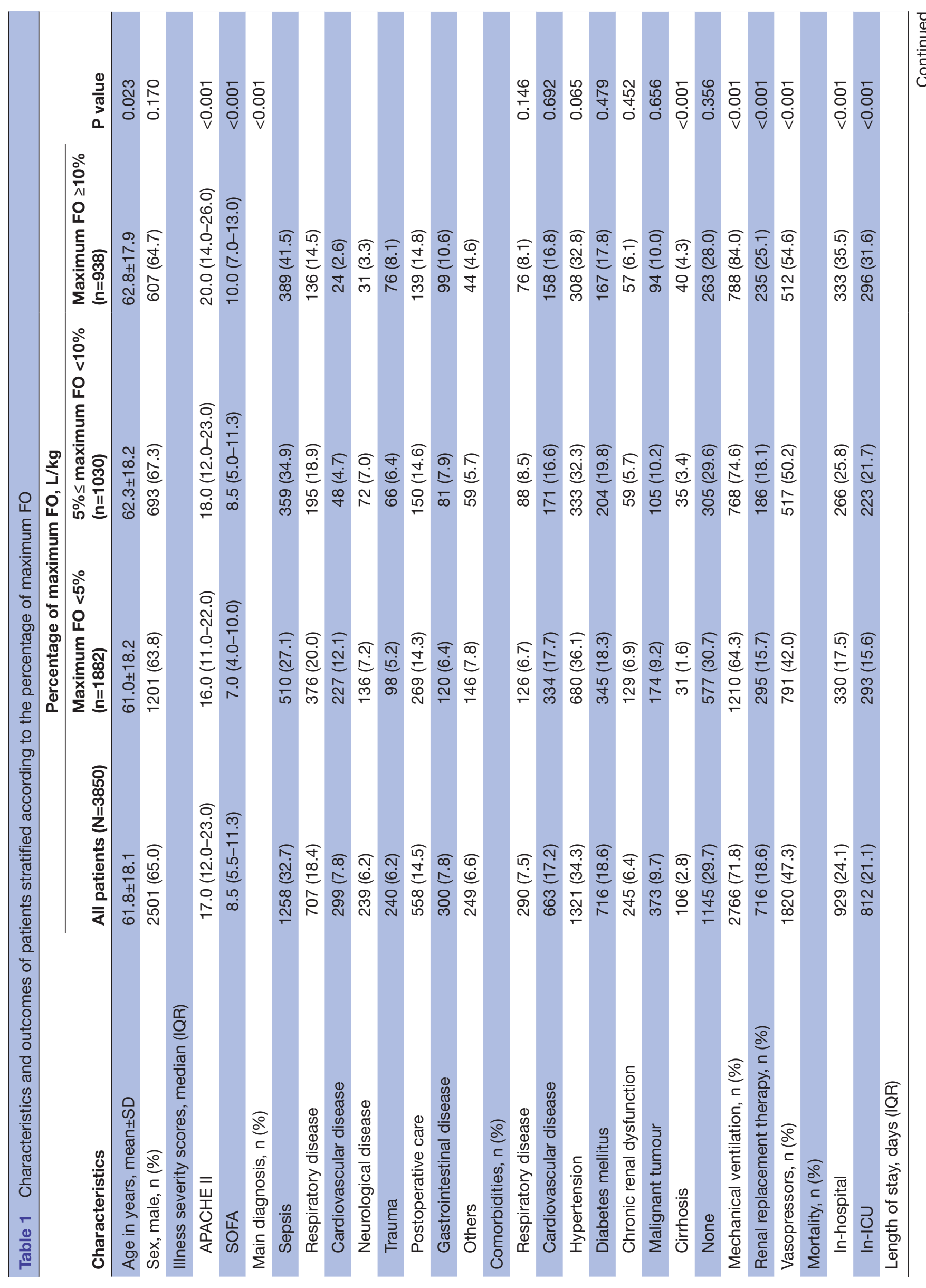


PSM analyses

The PSM method was used to match the patients with or without a maximum FO $\geq 5 \% \mathrm{~L} / \mathrm{kg}$, and 1078 (2156 patients) matched pairs were produced (online supplemental table S2). There was no significant difference in baseline characteristics, diagnosis on admission, comorbidities, and use of RRT and MV in matched study subjects. Every $1 \%$ increase in maximum FO was significantly associated with a $3 \%$ increase in risk of in-hospital mortality. Additionally, when maximum FO was entered as a categorical variable, a maximum FO greater than $10 \% \mathrm{~L} / \mathrm{kg}$ was associated with a $41 \%$ increased risk of in-hospital mortality compared with those who had a maximum FO lower than 5\% (online supplemental table S3).

In the dose-response analysis, we found similar associations between maximum FO and risk of in-hospital mortality with a nadir of $4.0 \% \mathrm{~L} / \mathrm{kg}$. Higher and lower maximum $\mathrm{FO}$ values were significantly associated with an increased risk of in-hospital mortality (figure 3B).

\section{DISCUSSION}

In this multicentre, prospective cohort study, we found that a higher level of maximum FO during the first 3 days of ICU admission was associated with an increased risk of in-hospital mortality in critically ill patients. Notably, we further demonstrated that this association was non-linear with a nadir of $2.4 \% \mathrm{~L} / \mathrm{kg}$, which indicated that maximum FO values greater than $5 \%$ or $10 \%$ and less than $0 \%$ were associated with an increased risk of in-hospital mortality. This study addressed that there might be an optimal 'plot' or 'range' of fluid balance for critically ill patients, which might be different for critically ill patients with different characteristics.

Fluid administration is an integral component of management of critically ill patients to maintain haemodynamic stability, organ function and tissue perfusion. Early fluid resuscitation has been shown to reverse tissue hypoperfusion and improve patient outcomes in several studies. ${ }^{21-23}$ However, several studies have supported the hypothesis that excessive fluid resuscitation may be harmful. ${ }^{4} 81324$ According to Alobaidi et al, ${ }^{3}$ a positive fluid balance in patients with severe bronchiolitis during the first 24 hours after admission to the paediatric intensive care unit (PICU) resulted in longer duration of PICU and hospital stays and longer duration of MV. However, fluid balance recorded during the second 24 hours $^{8}$ or within 72 hours ${ }^{13}$ after ICU admission, but not during the first 24 hours, is strongly associated with an increased risk of mortality. However, the studies described above did not explicitly define FO, but rather presented the association between positive fluid balance and outcomes.

Several clinical studies have reported that FO increases mortality in patients with sepsis, ${ }^{13} 25$ acute respiratory distress syndrome ${ }^{9}$ and AKI. ${ }^{10} 1124$ The Beijing Acute Kidney Injury Trial group ${ }^{11}$ and other researchers ${ }^{12} 26$ have considered FO as an accumulation of fluid balance greater than $10 \%$ of the body weight in kilograms and 
Table 2 Results of the Cox proportional hazard regression analysis of the risk of in-hospital mortality

Maximum FO as a continuous variable* $^{*}$

Maximum FO as a nominal variable

\begin{tabular}{|c|c|c|c|c|c|}
\hline & \multicolumn{2}{|l|}{ variable $^{*}$} & \multicolumn{3}{|c|}{ Maximum FO as a nominal variable } \\
\hline & HR $(95 \% \mathrm{Cl})$ & $P$ value & $\begin{array}{l}\text { Maximum FO <5\% } \\
(\mathrm{n}=1475) \\
\text { Reference }\end{array}$ & $\begin{array}{l}5 \% \leq \text { maximum FO } \\
<10 \%(n=773) \\
\text { HR }(95 \% \mathrm{Cl})\end{array}$ & $\begin{array}{l}\text { Maximum FO } \geq 10 \% \\
(\mathrm{n}=596) \\
\mathrm{HR}(95 \% \mathrm{Cl})\end{array}$ \\
\hline Model 1 & 1.06 (1.04 to 1.08$)$ & $<0.001$ & 1.000 & 1.38 (1.14 to 1.62$)$ & 2.11 (1.86 to 2.42$)$ \\
\hline Model 2 & 1.05 (1.03 to 1.07$)$ & $<0.001$ & 1.000 & $1.36(1.15$ to 1.61$)$ & 1.81 (1.56 to 2.11$)$ \\
\hline Model 3 & 1.04 (1.01 to 1.06$)$ & $<0.001$ & 1.000 & 1.30 (1.07 to 1.48$)$ & 1.63 (1.37 to 1.93$)$ \\
\hline Model 4 & 1.04 (1.03 to 1.05$)$ & $<0.001$ & 1.000 & $1.27(1.10$ to 1.52$)$ & 1.44 (1.25 to 1.67$)$ \\
\hline
\end{tabular}

Model 1: crude HR.

Model 2: adjusted for age, sex and APACHE II score.

Model 3: additionally adjusted for main diagnosis and comorbidities.

Model 4: additionally adjusted for time of maximum fluid overload, mechanical ventilation, renal replacement therapy and use of vasopressors.

${ }^{*} \mathrm{HR}$ was examined per $1 \%(\mathrm{~L} / \mathrm{kg})$ increase in maximum $\mathrm{FO}$.

APACHE II, Acute Physiology and Chronic Health Evaluation II; FO, fluid overload.

showed that FO was a risk factor for the incidence of AKI. In our study, critically ill patients tended to accumulate fluid starting on the day of ICU admission, and the deceased patients had accumulated much more fluid. In addition, as the volume of FO increased, the in-hospital mortality rate increased. Diminishing the effect of a negative fluid balance, the maximum FO might better explain the status of fluid accumulation in critically ill patients in the period of study. ${ }^{14}$ The FO aggrevate the underlying status of patients with AKI and maximum FO is an independent risk factor for the incidence of AKI and increases the severity of AKI. ${ }^{11}{ }^{14}$ In our study, maximum FO was an independent risk factor for in-hospital mortality (HR $1.04,95 \%$ CI 1.03 to 1.05 ), and for every $10 \mathrm{~mL} / \mathrm{kg}$ increase in maximum FO the risk of mortality increased by $4 \%$. A higher maximum FO resulted in a lower survival probability. Compared with patients presenting the lowest percentage of maximum FO (maximum $\mathrm{FO}<5 \%$ ), the risk of mortality increased 1.3 times in patients with a middle percentage of maximum FO $(5 \% \leq$ maximum $\mathrm{FO}<10 \%)$

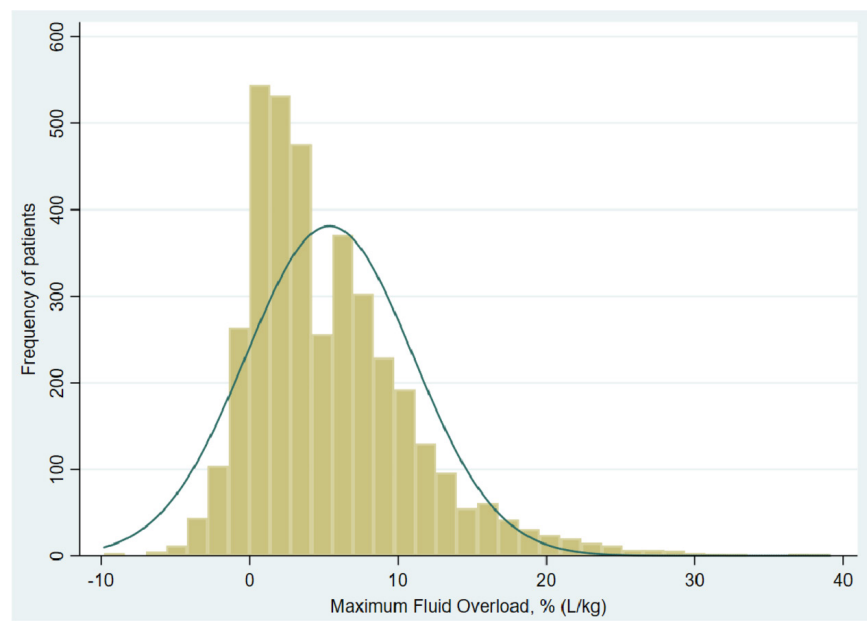

Figure 2 Distribution of the maximum fluid overload in all patients.
(HR $1.27,95 \%$ CI 1.10 to 1.52 ) and 1.4 times in patients with the highest percentage of maximum FO (HR 1.44, 95\% CI 1.25 to 1.67). Therefore, a maximum FO greater than $10 \%$ and between $5 \%$ and $10 \%$ both increased the risk of in-hospital mortality. This result was similar to that of a study on AKI in critically ill children that defined FO as CFB greater than $5 \%$ of the body weight on admission and found that $\mathrm{FO}$ was associated with an increased risk of AKI and PICU mortality. ${ }^{2}$

However, a study in the USA identified different FO cut-off values associated with in-hospital mortality. ${ }^{24}$ Garzotto and colleagues ${ }^{14}$ postulated that FO should not only be considered as a level greater than a fixed value but also any levels of positive fluid balance. Furthermore, their study showed that both positive and negative fluid balance might be associated with adverse outcomes. ${ }^{27} 28$ We used logistic regression models with RCS to better illustrate this dose-response relationship between FO and outcomes and observed a non-linear association between maximum FO and in-hospital mortality. Patients with a maximum $\mathrm{FO}$ of $2.4 \% \mathrm{~L} / \mathrm{kg}$ had the lowest risk of in-hospital mortality. These associations suggest that a higher maximum FO is significantly associated with an increased risk of in-hospital mortality. Although the sample size of patients with a maximum FO less than $0 \%$ was small, we still observed a slightly increased risk of in-hospital mortality. This finding appears to be logical. First, volume depletion causes hypovolaemia and tissue or organ hypoperfusion, which are associated with poor clinical outcomes. Second, patients who came from the emergency department, other wards or ICU, or postoperative patients, may have received fluid resuscitation prior to ICU admission and were non-fluid-responsive; these patients needed higher dose vasopressor to improve MAP and tissue hypoperfusion, which leads to adverse effects $^{29}{ }^{30}$ and higher mortality in patients with sepsis. ${ }^{31}$ However, we failed to consider fluid input and output prior to ICU admission or in the operating theatre, which 

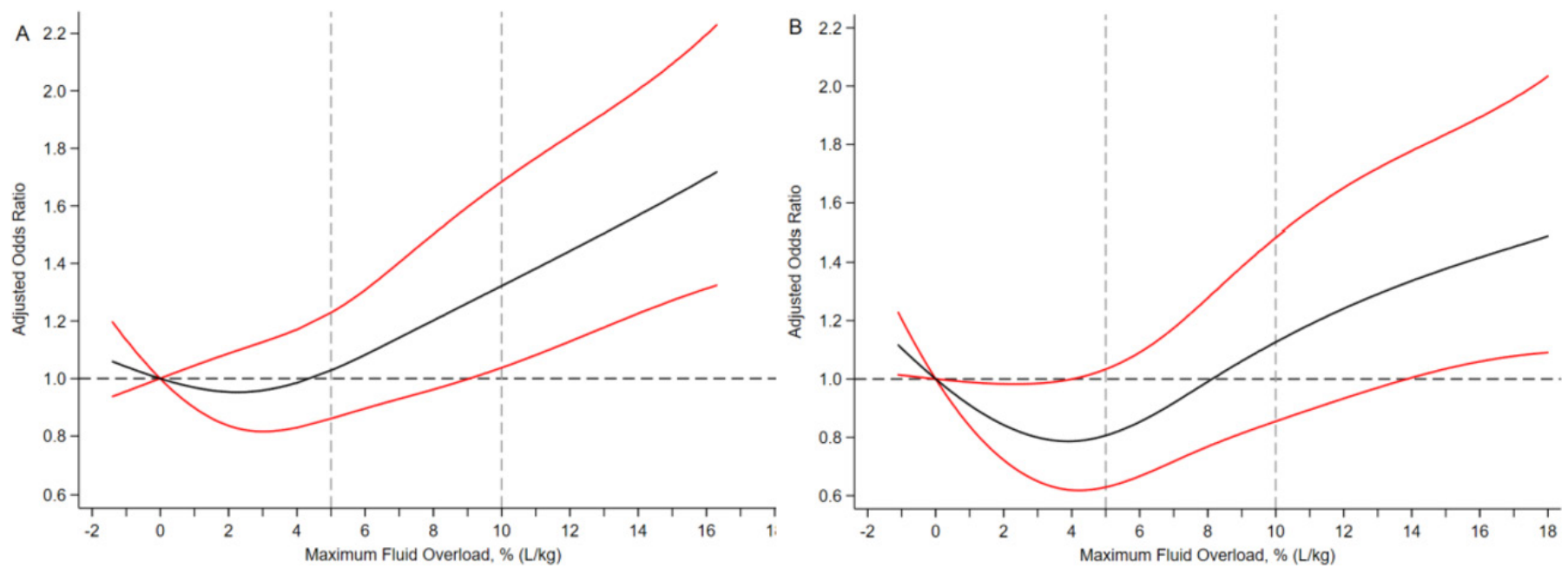

Figure 3 Adjusted OR between maximum fluid overload and in-hospital mortality (A) for all and (B) following propensity score matching. The black line indicates the adjusted OR, and the red lines indicate the $95 \% \mathrm{Cl}$ bands. The vertical grey dashed lines indicate $5 \%$ and $10 \% \mathrm{~L} / \mathrm{kg}$ of the maximum fluid overload. The reference for the maximum fluid overload is $0 \%$. Data were fitted using a multivariable logistic regression model with a restricted cubic spline with four knots (5th, 35th, 65th and 95th percentiles) for maximum fluid overload which was adjusted for potential confounders (model 4). Due to the small sample size, the lowest $5 \%$ and highest $5 \%$ of participants are not shown in the figures.

may affect outcomes, and our findings require future validation. In the sensitivity analysis, maximum FO was associated with an increased risk of in-hospital mortality in patients with or without shock, but patients with shock who needed more fluid resuscitation seemed to have a larger optimal range of fluid balance. When we used PSM to control for some potential confounders, the association between maximum FO and in-hospital mortality still persisted; that is, a 'spot' or 'range' for an optimal fluid balance may exist. This non-linear association has been reported in several studies. ${ }^{152728}$ Further studies are needed to confirm the precise margins of this 'spot' or 'range' of fluid balance in patients with acute critical illnesses.

There are several limitations to this study. First, the observational study design is not suitable to infer cause and effect. Second, although we used PSM to balance some important confounders, we may have failed to adjust for other potential confounders in our study. Third, we did not consider the fluid input and output before ICU admission or in the operating theatre, which cannot be ignored. Fourth, diuretic use and type of intravenous fluids were not documented, which may influence fluid output and outcomes. ${ }^{15}$ 32-34 Finally, we used maximum
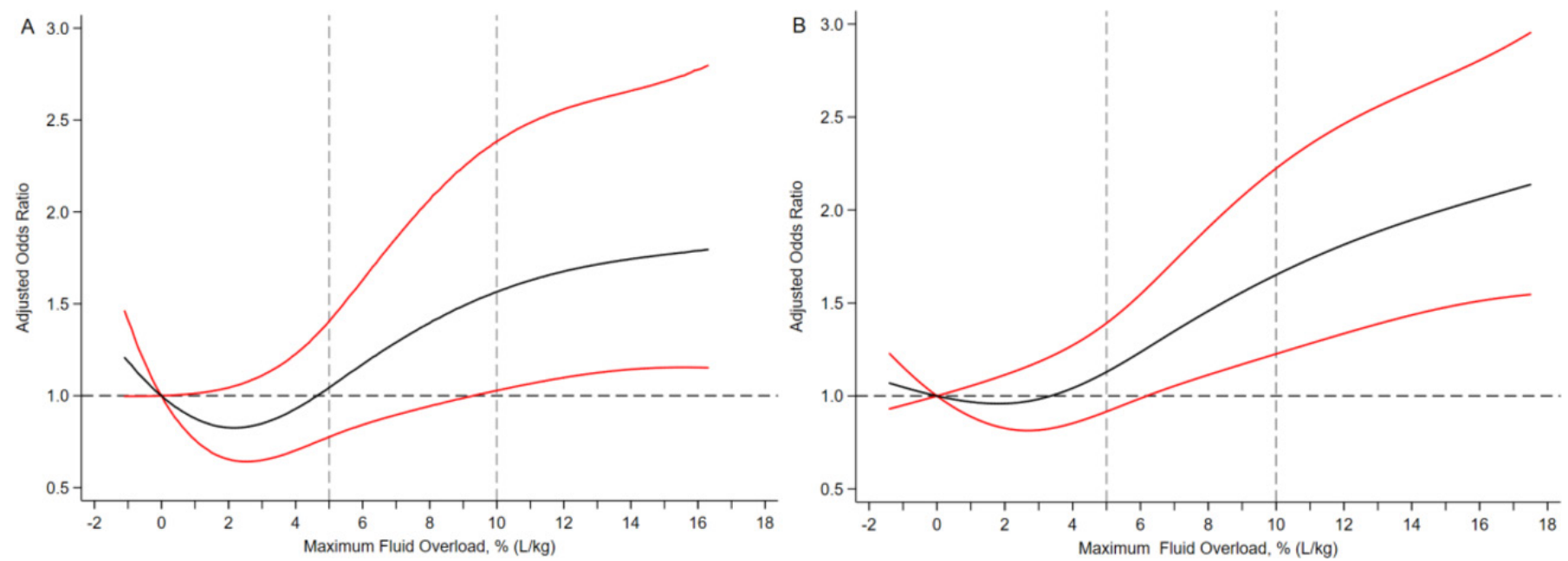

Figure 4 Adjusted OR between maximum fluid overload and in-hospital mortality in patients with (A) or without (B) shock. The black line indicates the adjusted OR, and the red lines indicate the $95 \% \mathrm{Cl}$ bands. The vertical grey dashed lines indicate $5 \%$ and $10 \% \mathrm{~L} / \mathrm{kg}$ of the maximum fluid overload. The reference for the maximum fluid overload is $0 \%$. Data were fitted using a multivariable logistic regression model with a restricted cubic spline with four knots (5th, 35th, 65th and 95th percentiles) for maximum fluid overload which was adjusted for potential confounders (model 4). Due to the small sample size, the lowest $5 \%$ and highest $5 \%$ of participants are not shown in the figures. 
FO to assess the degree of FO, but these values may not represent trends in fluid balance. We need to perform a latent growth model analysis to further explore the relationship between fluid balance and outcomes.

\section{CONCLUSIONS}

Our multicentre study revealed an association between FO and an increased risk of in-hospital mortality, and a non-linear association suggested that higher and lower fluid balance levels were associated with an increased risk of in-hospital mortality. However, fluid balance is accompanied by diverse risks of mortality in critically ill patients with or without shock. Further studies should be performed to explore the relationship between FO and mortality in patients with various diseases and determine optimal fluid management strategies.

\section{Author affiliations}

${ }^{1}$ Department of Epidemiology and Health Statistics, School of Public Health, Capital Medical University, Beijing, China

${ }^{2}$ Department of Critical Care Medicine, Fuxing Hospital, Capital Medical University, Beijing, China

${ }^{3}$ Department of Critical Care Medicine, Xuanwu Hospital, Capital Medical University, Beijing, China

${ }^{4}$ Department of General Practice, Beitaipingzhuang Community Health Service Centre, Beijing, China

${ }^{5}$ Medical Intensive Care Unit, Peking Union Medical College Hospital, Peking Union Medical College Hospital and Chinese Academy of Medical Sciences, Beijing, China

Acknowledgements We gratefully acknowledge the National Science and Technology Supporting Plan of the Ministry of Science and Technology of the People's Republic of China, a government fund used to improve healthcare quality and data collection, for providing financial support. We also acknowledge the following members of the CCCST workgroup who contributed data and samples and have enabled this study to be conducted: Bin Du, Medical Intensive Care Unit, Peking Union Medical College Hospital, Beijing, China; Li Weng, Medical Intensive Care Unit, Peking Union Medical College Hospital, Beijing, China; Tong Li, Department of Critical Care Medicine, Beijing Tongren Hospital, Capital Medical University, Beijing, China; Meili Duan, Department of Critical Care Medicine, Beijing Friendship Hospital, Capital Medical University, Beijing, China; Wenxiong Li, Surgical Intensive Care Unit, Beijing Chaoyang Hospital, Capital Medical University, Beijing, China; Bing Sun, Department of Respiratory and Critical Care Medicine, Beijing Institute of Respiratory Medicine, Beijing Chaoyang Hospital, Capital Medical University, Beijing, China; Jianxin Zhou, Department of Critical Care Medicine, Beijing Tiantan Hospital, Capital Medical University, Beijing, China; Jianguo Jia, Surgical Intensive Care Unit, Xuanwu Hospital, Capital Medical University, Beijing, China; Xi Zhu, Department of Critical Care Medicine, Peking University Third Hospital, Beijing, China; Qingyuan Zhan, Department of Critical Care Medicine, China-Japan Friendship Hospital, Beijing, China; Xiaochun Ma, Department of Critical Care Medicine, The First Affiliated Hospital of China Medical University, Shenyang, China; Tiehe Qin and Shouhong Wang, Department of Critical Care Medicine, Guangdong Geriatric Institute, Guangdong General Hospital, Guangdong, China; Yuhang Ai, Department of Critical Care Medicine, Xiangya Hospital, Central South University, Changsha, China; Yan Kang and Xuelian Liao, Department of Critical Care Medicine, West China Hospital, Sichuan University, Sichuan, China; Xiangyuan Cao, Department of Critical Care Medicine, General Hospital of Ningxia Medical University, Ningxia, China; Yushan Wang, Intensive Care Unit, The First Hospital of Jilin University, Changchun, China; and Duming Zhu, Surgical Intensive Care Unit, Department of Anaesthesiology, ZhongShan Hospital, FuDan University, Shanghai, China. The CCCST group performed the recruitment and data collection.

Contributors XX conceived, designed and led the study. MW, BZ and LJ were involved in the design of the study and analysis of the data. YW, WenL, GL, WeiL and JW finalised the analysis and interpreted the findings. MW wrote the drafts of the manuscript. BD, YH and XX commented on and helped revise drafts of the manuscript. All authors contributed to this article and all have approved the final manuscript.
Funding This study was supported by the National Science and Technology Supporting Plan of the Ministry of Science and Technology of the People's Republic of China (2012BAl11B05). The funder had no role in the design and conduct of the study; management, analysis and interpretation of the data; preparation of the manuscript; or the decision to submit for publication.

Competing interests None declared.

Patient consent for publication Not required.

Ethics approval The study protocol was approved by the ethics committees of Fuxing Hospital, Capital Medical University (approval notice number 2013FXHEC-KY018) and all other centres.

Provenance and peer review Not commissioned; externally peer reviewed.

Data availability statement The datasets generated and or analysed during the current study are available from the corresponding author on reasonable request.

Supplemental material This content has been supplied by the author(s). It has not been vetted by BMJ Publishing Group Limited (BMJ) and may not have been peer-reviewed. Any opinions or recommendations discussed are solely those of the author(s) and are not endorsed by BMJ. BMJ disclaims all liability and responsibility arising from any reliance placed on the content. Where the content includes any translated material, BMJ does not warrant the accuracy and reliability of the translations (including but not limited to local regulations, clinical guidelines, terminology, drug names and drug dosages), and is not responsible for any error and/or omissions arising from translation and adaptation or otherwise.

Open access This is an open access article distributed in accordance with the Creative Commons Attribution Non Commercial (CC BY-NC 4.0) license, which permits others to distribute, remix, adapt, build upon this work non-commercially, and license their derivative works on different terms, provided the original work is properly cited, appropriate credit is given, any changes made indicated, and the use is non-commercial. See: http://creativecommons.org/licenses/by-nc/4.0/.

ORCID iD

Meiping Wang http://orcid.org/0000-0003-3838-6204

\section{REFERENCES}

1 El Khuri C, Abou Dagher G, Chami A, et al. The impact of EGDT on sepsis mortality in a single tertiary care center in Lebanon. Emerg Med Int 2019;2019:1-8.

2 Li Y, Wang J, Bai Z, et al. Early fluid overload is associated with acute kidney injury and PICU mortality in critically ill children. Eur J Pediatr 2016;175:39-48.

3 Alobaidi R, Morgan C, Basu RK, et al. Association between fluid balance and outcomes in critically ill children: a systematic review and meta-analysis. JAMA Pediatr 2018;172:257-68.

4 Flores-Gonzalez JC, Valladares CM, Yun Castilla C, et al. Bronquiolitis en La Unidad de Cuidados Intensivos Pediatricos W: association of fluid overload with clinical outcomes in critically ill children with bronchiolitis: Bronquiolitis en La Unidad de Cuidados Intensivos Pediatricos (BRUCIP) study. Pediatr Crit Care Med 2019;20:e130-6.

5 Casas-Aparicio GA, León-Rodríguez I, Hernández-Zenteno RdeJ, et al. Aggressive fluid accumulation is associated with acute kidney injury and mortality in a cohort of patients with severe pneumonia caused by influenza A H1N1 virus. PLoS One 2018;13:e0192592.

6 Chaudhari AP, Pavan M, Mehta HJ. Influence of fluid balance on morbidity and mortality in critically ill patients with acute kidney injury. Iran J Kidney Dis 2016;10:177-81.

7 Kissoon NR, Mandrekar JN, Fugate JE, et al. Positive fluid balance is associated with poor outcomes in subarachnoid hemorrhage. $J$ Stroke Cerebrovasc Dis 2015;24:2245-51.

8 Shen Y, Ru W, Huang X, et al. Time-Related association between fluid balance and mortality in sepsis patients: interaction between fluid balance and haemodynamics. Sci Rep 2018;8:10390.

9 Zinter MS, Spicer AC, Liu KD, et al. Positive cumulative fluid balance is associated with mortality in pediatric acute respiratory distress syndrome in the setting of acute kidney injury. Pediatr Crit Care Med 2019;20:323-31.

10 Codes L, de Souza YG, D'Oliveira RAC, et al. Cumulative positive fluid balance is a risk factor for acute kidney injury and requirement for renal replacement therapy after liver transplantation. World $J$ Transplant 2018;8:44-51.

11 Wang N, Jiang L, Zhu B, et al. Fluid balance and mortality in critically ill patients with acute kidney injury: a multicenter prospective epidemiological study. Crit Care 2015;19:371. 
12 Fülöp T, Pathak MB, Schmidt DW, et al. Volume-related weight gain and subsequent mortality in acute renal failure patients treated with continuous renal replacement therapy. Asaio J 2010;56:333-7.

13 Sakr Y, Rubatto Birri PN, Kotfis K, et al. Higher fluid balance increases the risk of death from sepsis: results from a large international audit. Crit Care Med 2017;45:386-94.

14 Garzotto F, Ostermann M, Martín-Langerwerf D, et al. The dose response multicentre investigation on fluid assessment (DoReMIFA) in critically ill patients. Crit Care 2016;20:196.

15 Woodward CW, Lambert J, Ortiz-Soriano V, et al. Fluid overload associates with major adverse kidney events in critically ill patients with acute kidney injury requiring continuous renal replacement therapy. Crit Care Med 2019;47:e753-60.

16 Knaus WA, Draper EA, Wagner DP, et al. Apache II: a severity of disease classification system. Crit Care Med 1985;13:818-29.

17 Vincent JL, de Mendonca A, Cantraine F, et al. Use of the SOFA score to assess the incidence of organ dysfunction/failure in intensive care units: results of a multicenter, prospective study. Critical Care Medicine 1998;26:1793-800.

18 Rhee C, Dantes R, Epstein L, et al. Incidence and trends of sepsis in US hospitals using clinical vs claims data, 2009-2014. JAMA 2017;318:1241-9.

19 Singer M, Deutschman CS, Seymour CW, et al. The third International consensus definitions for sepsis and septic shock (Sepsis-3). JAMA 2016;315:801-10.

20 Desquilbet L, Mariotti F. Dose-Response analyses using restricted cubic spline functions in public health research. Stat Med 2010;29:1037-57.

21 Rivers E, Nguyen B, Havstad S, et al. Early goal-directed therapy collaborative G: early goal-directed therapy in the treatment of severe sepsis and septic shock. N Engl J Med 2001;345:1368-77.

22 Rhodes A, Evans LE, Alhazzani W, et al. Surviving sepsis campaign: international guidelines for management of sepsis and septic shock: 2016. Crit Care Med 2017:45:486-552.

23 Loflin R, Winters ME. Fluid resuscitation in severe sepsis. Emerg Med Clin North Am 2017;35:59-74
24 Neyra JA, Li X, Canepa-Escaro F, et al. Acute kidney injury in critical illness study G: cumulative fluid balance and mortality in septic patients with or without acute kidney injury and chronic kidney disease. Crit Care Med 2016;44:1891-900.

25 Márquez-González H, Casanova-Bracamontes L, Muñoz-Ramírez $\mathrm{CM}$, et al. Relation between fluid overload and mortality in children with septic shock. Arch Argent Pediatr 2019;117:105-13.

26 Bouchard J, Soroko SB, Chertow GM, et al. Program to improve care in acute renal disease study G: fluid accumulation, survival and recovery of kidney function in critically ill patients with acute kidney injury. Kidney Int 2009;76:422-7.

27 Myles PS, Bellomo R, Corcoran T, et al. Restrictive versus liberal fluid therapy for major abdominal surgery. $N$ Engl J Med 2018;378:2263-74.

28 Balakumar V, Murugan R, Sileanu FE, et al. Both positive and negative fluid balance may be associated with reduced long-term survival in the critically ill. Crit Care Med 2017;45:e749-57.

29 Brown SM, Lanspa MJ, Jones JP, et al. Survival after shock requiring high-dose vasopressor therapy. Chest 2013;143:664-71.

30 Lesur O, Delile E, Asfar P, et al. Hemodynamic support in the early phase of septic shock: a review of challenges and unanswered questions. Ann Intensive Care 2018;8:102.

31 Roberts RJ, Miano TA, Hammond DA, et al. Observation of variation in F Lais-CovRiSSG, network SD: evaluation of vasopressor exposure and mortality in patients with septic shock. Crit Care Med 2020

32 Barbar SD, Clere-Jehl R, Bourredjem A, et al. Timing of renalreplacement therapy in patients with acute kidney injury and sepsis. N Engl J Med 2018;379:1431-42.

33 Barea-Mendoza J, Chico-Fernández M, Montejo-González JC. Balanced crystalloids versus saline in critically ill adults. N Engl J Med 2018;378:1950-1.

34 Hammond DA, Lam SW, Rech MA, et al. Balanced crystalloids versus saline in critically ill adults: a systematic review and metaanalysis. Ann Pharmacother 2020;54:5-13. 